\title{
Nanocrystal emitters for enhanced photovoltaics in UV
}

\author{
Evren Mutlugun, Ibrahim Murat Soganci, and Hilmi Volkan Demir \\ Department of Physics, Department of Electrical and Electronics Engineering, Nanotechnology Research Center, and \\ Institute of Materials Science and Nanotechnology, Bilkent University, Ankara, 06800 Turkey \\ Tel:[+90](312) 290-1021 E-mail: volkan@bilkent.edu.tr
}

Because of its mature processing technology, silicon has been commonly used in electronics including integrated circuits (e.g., CMOS) and in optoelectronics including CCD cameras, photodetectors, and photovoltaics [1-4]. Furthermore, silicon photonics has recently made significant progress towards the ultimate goal of realizing optoelectronic devices on integrated circuits. However, Si material is constrained in different aspects for use in optoelectronics. One of such limitations is observed in its low responsivity in the ultraviolet (UV) spectral range, limiting the operating range of Si based optoelectronic devices only across the visible and near infrared. As a result, in photovoltaic applications, Si based solar cells are limited for use in UV. Therefore, for UV operation, there is a need for the enhancement of Si based optoelectronic devices [5,6]. To this end in this work, we proposed and demonstrated nanocrystal hybridization of silicon based solar cells for enhanced photovoltaics in the UV part of the light spectrum, which is critically important since the UV range makes up around $12 \%$ of the entire solar spectrum.

Semiconductor nanocrystals, typically with radii of few nanometers, exhibit strongly size-dependent optical emission, absorption, and excitation properties. These size-tunable properties of nanocrystals enable us to develop nanocrystal scintillators tuned for use on a silicon platform. For this purpose, we used highly luminescent $\mathrm{CdSe} / \mathrm{ZnS}$ core-shell nanocrystals. These nanocrystals were designed to match the high responsivity region of the silicon. They have emission at around $548 \mathrm{~nm}$ in film and have high absorption coefficient in UV, as presented in Fig. 1 (left). By wavelength up-converting of incident UV radiation to visible emission using these nanocrystal emitters, we demonstrated hybrid nanocrystal-silicon devices with the enhanced UV responsivity in silicon based solar cells. The responsivity of the bare solar cell was demonstrated to be enhanced up to 10 folds in the UV as shown in Fig. 1 (right).
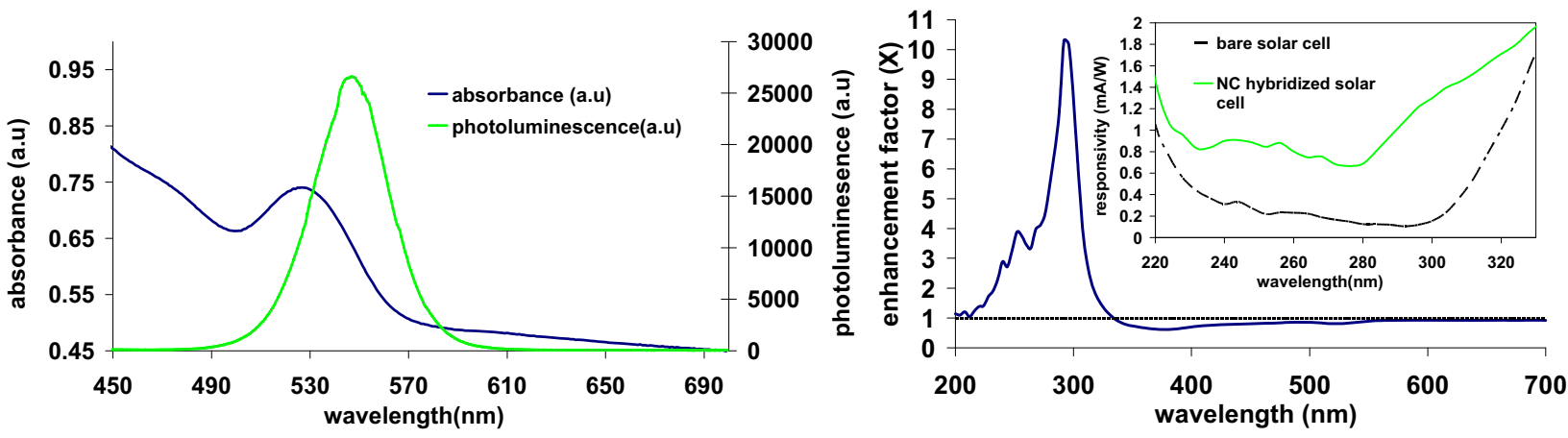

Figure 1. Photoluminescence and absorbance characteristics of our green emitting nanocrystals (left) and the enhancement in the responsivity of Si solar cell hybridized with nanocrystals with respect to the bare solar cell, where the inset shows the responsivity of the bare solar cell and NC hybridized solar cell in the UV region of the light spectrum, respectively (right).

In operation, the incident UV photons are first absorbed by the nanocrystal scintillators, which in turn luminescence in the visible, subsequently incident on to the integrating solar cell underneath. Since the Si solar cell features high responsivity in the visible region as opposed to the UV, these up-converted photons are then strongly absorbed by the solar cell, contributing to the solar energy conversion. In this work we characterized the solar cell parameters as a function of the amount of hybridized nanocrystals. The solar cell parameters including open circuit voltage, short circuit current, fill factor, and solar conversion efficiency were shown to be enhanced by hybridizing nanocrystal emitters on the silicon solar cell, as listed in Table 1. The characterization of the solar cell was performed using a Xe lamp, which provides similar UV content as in the solar spectrum. Figure 2 (left) shows the modification of the I-V characteristics of the solar cell with the various amounts of hybridized nanocrystals under identical illumination conditions. As the amount of hybridized nanocrystals was varied from $7.78 \mathrm{nmol}$ to $152.10 \mathrm{nmol}$, we observed the enhanced solar cell parameters converging to certain saturation levels (Fig. 2 (right)). Beyond these saturation 
levels, further enhancements were not observed. In this characterization, a maximum of 2.11 fold enhancement in the solar conversion efficiency was achieved. This improvement was attributed to the extension of solar cell responsivity towards UV, better impedance matching to the solar cell, and possibly multiple exciton formation under high photon energy excitation.
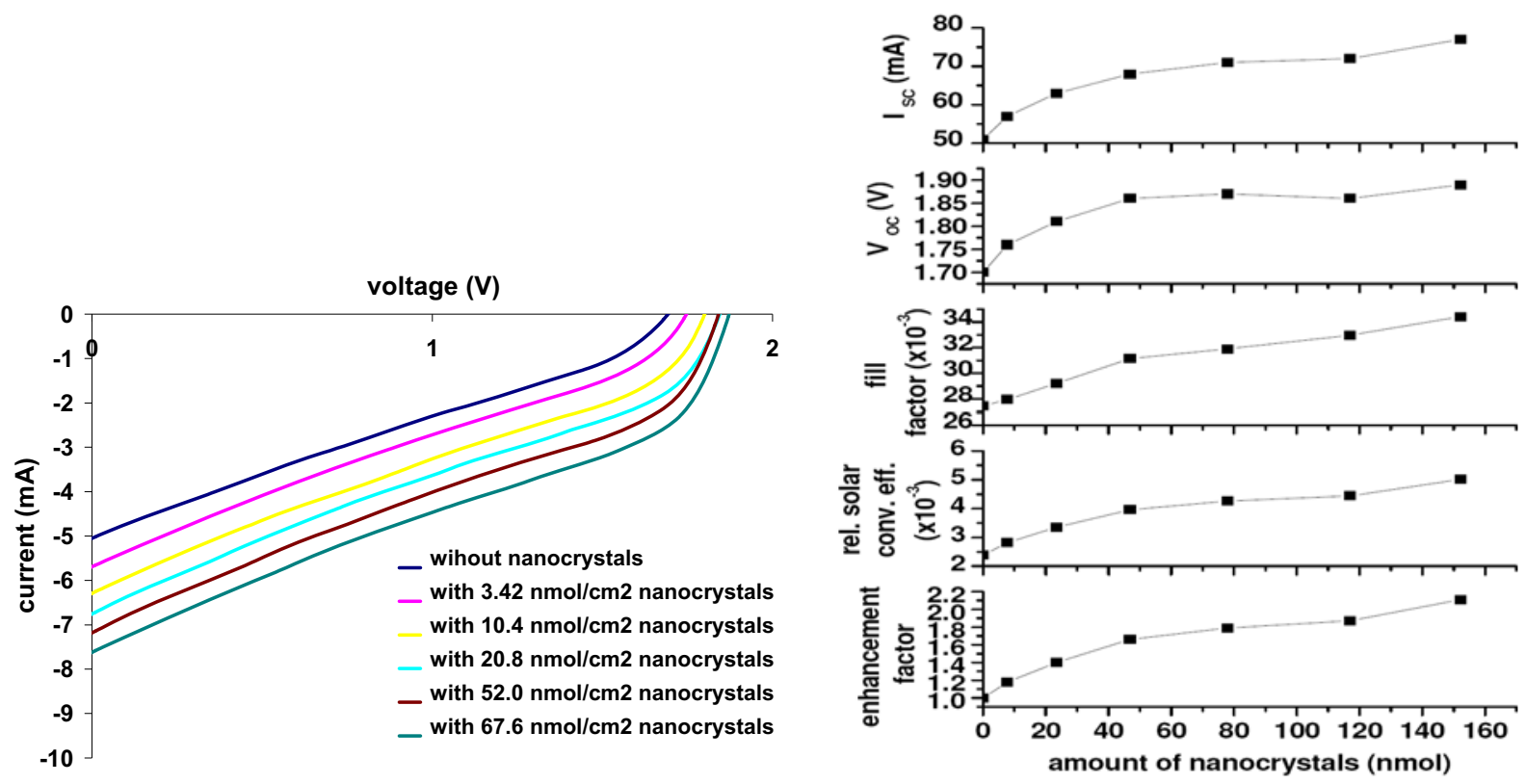

Figure 2. I-V characteristics of the solar cell in the $4^{\text {th }}$ quadrant, characterized with respect to the changing amount of hybridized nanocrystals under $\mathrm{Xe}$ illumination (left), and the resulting solar cell parameters and the corresponding enhancement factor as a function of the amount of nanocrystals (right).

\begin{tabular}{l|r|r|r|r|r|r|r} 
Amount of nanocrystals (nmol) & None & 7.78 & 23.40 & 46.80 & 77.85 & 117.00 & 152.10 \\
\hline$(\mathrm{I} \times \mathrm{V})_{\max }(\mathrm{A} \times \mathrm{V})\left(\times 10^{-3}\right)$ & 2.38 & 2.82 & 3.35 & 3.96 & 4.26 & 4.44 & 5.01 \\
$\mathrm{I}_{\mathrm{sc}} \times \mathrm{V}_{\mathrm{oc}}(\mathrm{A} \times \mathrm{V})\left(\times 10^{-3}\right)$ & 8.67 & 10.09 & 11.47 & 12.72 & 13.35 & 13.46 & 14.60 \\
Fill factor $\left(\times 10^{-2}\right)$ & 27.45 & 27.95 & 29.21 & 31.14 & 31.91 & 32.97 & 34.40 \\
Relative solar conversion efficiency & 2.38 & 2.82 & 3.35 & 3.96 & 4.60 & 4.44 & 5.01 \\
Enhancement factor & 1.00 & 1.18 & 1.40 & 1.66 & 1.79 & 1.87 & 2.11
\end{tabular}

Table. 1. Solar cell parameters for different amounts of hybridized nanocrystal emitters.

In conclusion, in our proof-of-concept demonstrations, we observed that nanocrystal hybridized solar cells provide the ability to enhance the solar cell parameters in UV. We demonstrated a 2-fold enhancement in the solar conversion efficiency and a 10-fold enhancement in the responsivity across the UV range of the solar spectrum. We are currently working on further improvements since the enhancement obtained by the hybridization of such nanocrystal scintillators also depends on engineering the quantum efficiency of these nanocrystal emitters in film. We believe that these results are promising for extending solar cell operation towards UV, which would also possibly offer a commercial value both in space and roof-top applications.

Acknowledgements: This work is supported by EU MOON, EU NOE PHOREMOST and TUBITAK 106E020, 104E114, 107E088, 107E297, 105E065, and 105E066. HVD also acknowledges support from Turkish Academy of Sciences (TUBA GEBIP) and European Science Foundation (ESF EURYI).

References

[1] O. M. Nayfeh, S. Rao, A. Smith, J. Therrien, and M. H. Nayfeh, IEEE Photonics Technology Letters, vol. 16, no. 8 (2004).

[2] C.B. Honsberg, "Approaches for Ultra-High Efficiency Solar Cells".

[3] N. Lalic, "Light emitting devices based on silicon nanostructures," KTH Royal Institute of Technology, Stockholm (2000).

[4] A. Pauchard, A. Rochas, Z. Randzelovic, P. A. Besse, and R. S. Popovic, IEDM Tech. Dig. 709-712 (2000).

[5] E. Mutlugun, I. M. Soganci, and H. V. Demir, Opt. Express 15, 1128-1134 (2007).

[6] E. Mutlugun, I. M. Soganci, and H. V. Demir, Opt. Express 16, 3537-3545 (2008). 